\title{
Financial Capacity and Illiteracy: Does Education Matter in Amnestic Mild Cognitive Impairment?
}

\author{
Vaitsa Giannouli* and Magda Tsolaki \\ School of Medicine, Aristotle University of Thessaloniki, Thessaloniki, Greece
}

Accepted 21 August 2021

Pre-press 2 September 2021

\begin{abstract}
Neuropsychological assessment in amnestic mild cognitive impairment (aMCI) becomes complicated when education-literacy is taken into consideration. This study sought to explore the potential influence of literacy/illiteracy and education on financial capacity in patients with multiple-domain aMCI. Six groups consisting of aMCI (illiterate-no formal education, literate with low education, and literate with high education) and non-demented controls were examined. Literacy has an effect on financial capacity, as the illiterate aMCI group alone had the lowest scores in a financial capacity test resembling the performance of patients with mild Alzheimer's disease. In controls there was a similar pattern, but all three healthy groups regardless of education scored above the cut-off score for incapacity.
\end{abstract}

Keywords: Amnestic mild cognitive impairment, financial capacity, literacy/illiteracy

\section{INTRODUCTION}

Illiterates represent a significant proportion of the world's population, and illiteracy has been supported to be associated with higher risk of prevalent and incident dementia [1]. However, so far there is no research in patients diagnosed with amnestic mild cognitive impairment (aMCI) and the possible influence that literacy/illiteracy and varying levels of education may have on a specific form of instrumental activity of daily living with cognitive components; patients' financial capacity. Loss of financial capacity is a common consequence of Alzheimer's disease, but also of Parkinson's disease, vascular dementia, and aMCI with and without comorbid depression [2-4]. The relationship between education and financial skills is not thoroughly examined, but it is likely that some

\footnotetext{
*Correspondence to: Vaitsa Giannouli, School of Medicine, Aristotle University of Thessaloniki, 54124, Thessaloniki, Greece. E-mail: giannouliv@ hotmail.com.
}

individuals due to lack of education may have never obtained a high degree of numeracy as a study so far supports for the role of numeracy as a significant risk factor for financial exploitation [5]. This research gap is prevalent not only in Greece [6], where older adults and older patients do not necessarily have a minimum formal education in elementary school, but also in many other countries worldwide in which individuals did not attend school for social (e.g., absence of schools during or immediately after World War II), and/or personal (e.g., learning difficulties) reasons [7].

Financial capacity in patients with aMCI is linked to impairments that differentiate them from healthy controls across a range of financial abilities [8], and more specifically semantic arithmetic knowledge; and to a lesser extent, visual memory and attention are reported as the best cognitive predictors of financial skill decline in this group of patients [9]. Moreover, comorbid depression has a detrimental influence on financial capacity in aMCI [4], while changes in the 
volume of brain areas such as left angular gyrus and amygdala are reported as strongly correlated to financial capacity deficits in aMCI [10].

Financial capacity is considered to be the single best predictor of capacity for independent living in older adults [3]. Although it is complex, as it comprises a variety of activities and specific skills, including performance skills (such as arithmetic counting coins/currency, paying bills etc.) and judgment-decision making skills [3], no systematic research on education and illiteracy effects has been reported so far. The aim of this study is to examine and compare multiple-domain aMCI patients' financial capacity performance to healthy older adults with the same demographic characteristics when literacy/illiteracy and years of education is serving as the grouping variable.

\section{METHODS}

One hundred twenty Greek older adults $(80 \%$ women, all right-handed) took part in the study as part of their neuropsychological assessment at the Memory Clinic of Papanikolaou General Hospital and elderly day care centers (as in similar studies [2-4]). Patients with aMCI were diagnosed using criteria for two clinical subtypes: 1) single-domain aMCI in which patients manifest isolated episodic memory impairments, and 2) multiple-domain aMCI with episodic memory impairments and deficits in one or more other cognitive domains. Patients with aMCI were selected according to Petersen criteria [11] and more specifically the diagnosis of multipledomain aMCI subtype, an impairment in memory and at least one other neuropsychological domain were present. The reason for focusing only on multipledomain aMCI was due to the small sample size of single-domain aMCI participants that rendered risky any statistical analyses $(\mathrm{N}=14)$. No depressive symptoms according to the 15-item Geriatric Depression Scale when a cut-off $6 / 7$ point which is applied for diagnosing depression were found with all participants scoring below 7 [12]. The multiple-domain aMCI patient group $(\mathrm{N}=60)$ was further divided in three subgroups, namely: 1) illiterate-no formal education, 2) literate with low education-up to 9 years basic education, and 3) literate with high educationmore than 9 years of compulsory education. The non-demented controls $(\mathrm{N}=60)$ were accordingly divided in three subgroups: 4) illiterate-no formal education, 5) literate with low education, and 6) lit- erate with high education. Literacy was self-reported based on the question: Did you ever learn to read or write? and If yes, how many years did your education last? The community-dwelling elders (control group) came from a larger subject pool of participants and were free of a diagnosis related to cognitive decline (all of them went through a neuropsychological assessment which was part of the screening process for a previous study [13]). They were approached following purposeful sampling (regarding their gender, age, and education characteristics).

Matching was done on a one-to-one basis, as the patients and healthy controls were matched individually. The healthy participants were matched with the patient group regarding their age $[\mathrm{F}(5,119)=1.190$, $p=0.318]$, and gender $\left[\chi^{2}(5)=3.125, p=0.681\right]$, in order to eliminate a possible influence of these variables as confounders. All participants had prior financial experience in real-life financial transactions. We employed as in other studies [2-4] explicit exclusion criteria for all participants: known history of stroke, traumatic brain injury and related neurosurgical interventions, a history of substance abuse, concomitant serious medical illness (such as significant visual and/or auditory impairment not corrected sufficiently by visual/auditory aids, a history of other neurologic or psychiatric disorder that could account for the patient's symptoms).

Written informed consent was obtained. The study was approved by the Ethics Committee of the Aristotle University of Thessaloniki (as part of a larger study [13]), and it was performed according to the declaration of Helsinki.

The participants' cognitive deficits were assessed with the Mini-Mental State Examination (MMSE) and in the case of illiterate participants with the equivalent Greek form of the modified version of MMSE, Hindi Mental State Examination (HMSE). In Greece, both tests are considered as comparable and equal in administration and analyses as they both measure global cognition and the total score if all items are answered is 30 points [14]. Additionally, participants underwent a detailed neuropsychological battery covering a variety of cognitive areas (presented in detail in [8]), and depressive symptomatology was assessed with the GDS-15. Financial capacity was assessed with the Greek version of the Legal Capacity for Property Law Transactions Assessment Scale (LCPLTAS) [13]. The LCPLTAS consists of seven main domains: 1) basic monetary skills, 2) cash transactions, 3) bank statement management, 4) bill payment, 5) financial conceptual knowledge, 6) financial decision 
Table 1

Demographic characteristics and MMSE/HMSE and LCPTLAS full scores in multiple-domain aMCI patients and healthy individuals (illiterate-no formal education, literate with low education and literate with high education)

\begin{tabular}{llllllll}
\hline Variable & $\begin{array}{l}\text { aMCI high } \\
\text { education }\end{array}$ & $\begin{array}{l}\text { aMCI low } \\
\text { education }\end{array}$ & $\begin{array}{l}\text { aMCI } \\
\text { illiterate* }\end{array}$ & $\begin{array}{l}\text { HC high } \\
\text { education }\end{array}$ & $\begin{array}{l}\text { HC low } \\
\text { education }\end{array}$ & $\begin{array}{l}\text { HC } \\
\text { illiterate* }\end{array}$ & $p$ \\
Age & 67.30 & 70.35 & 67.35 & 68.95 & 66.40 & 71.30 & 0.318 \\
& $(8.24)$ & $(6.15)$ & $(6.68)$ & $(8.80)$ & $(4.89)$ & $(11.06)$ & 0.00 \\
Education (y) & 15.35 & 4.95 & 0.00 & 19.03 & 4.95 & $(0.050$ \\
MMSE-HMSE* & $(1.03)$ & $(0.60)$ & $(0.00)$ & $(4.40)$ & $(0.60)$ & $(0.00)$ & 28.25 \\
& 27.80 & 26.55 & 25.60 & 29.20 & 28.80 & $(1.65)$ & $<0.001$ \\
LCPTLAS full total & $(1.05)$ & $(0.94)$ & $(1.14)$ & $(0.52)$ & $(0.61)$ & 195.25 & $<0.001$ \\
score & 195.29 & 142.31 & 110.85 & 211.55 & 199.20 & $(25.01)$ & \\
\hline
\end{tabular}

MMSE, Mini-Mental State Examination; HMSE, Hindi Mental State Examination; LCPTLAS, Legal Capacity for Property Law Transactions Assessment Scale; aMCI, multiple-domain amnestic mild cognitive impairment; HC, healthy controls.

making, and 7) knowledge of personal assets [13]. The LCPLTAS has a cutoff score corresponding to maximum sensitivity and maximum specificity the scores 211 and 154 respectively, while the cutoff that optimizes both based on Youden's index is 200 (range of scores 0 to 212 points) [13]. Although there exists both an extended as well as a brief version of LCPLTAS, in this study only the extended version was used as this form is considered to give richer information regarding different facets of financial capacity and is in use in clinical practice in Greece.

\section{Statistics}

One-way analyses of variance (ANOVAs) were conducted in order to examine demographic factors and cognitive test scores of the six groups [multiple-domain aMCI (1) illiterate-no formal education, 2) literate with low education, and 3) literate with high education), and non-demented controls (4) illiterate-no formal education, 5) literate with low education, and 6) literate with high education)]. In addition to that, correlations between years of education, age, MMSE-HMSE scores, sex, and LCPTLAS were performed for the healthy group as well as the multiple-domain aMCI group. A regression analysis was performed with education, age, and MMSE-HMSE scores as independent variables and LCPTLAS score as the dependent variable. Only raw scores were used regarding LCPTLAS performance. Probability values $<0.05$ (two-tailed) were considered statistically significant.

\section{RESULTS}

A strong positive correlation was found MMSE score and LCPTLAS $(r=0.998, p<0.043)$, but no correlations were found between years of educa- tion and LCPTLAS $(r=0.998, p=0.360)$, between sex and LCPTLAS (point biserial correlation coefficient, $\mathrm{r}_{\mathrm{pbis}}=-0.110, p=0.418$ ), and for age and LCPTLAS ( $\mathrm{r}=-0.160, p=0.898$ ), for the multipledomain aMCI group. For the healthy group, no correlations were found between age and LCPTLAS $(\mathrm{r}=-0.209, p=0.866)$, MMSE and LCPTLAS $(\mathrm{r}=0.929, p=0.242)$, and sex (point biserial correlation coefficient, $\left.r_{\text {pbis }}=-0.149, p=0.257\right)$, but a strong statistical positive correlation was found between the years of formal education and LCPTLAS $(r=0.980, p=0.012)$. Linear Regression model, "Enter" method (in the total sample) indicated that education $(\mathrm{B}=-0.203, \mathrm{SE}=1.274, p=0.888)$, age $(\mathrm{B}=-0.586, \mathrm{SE}=1.711, p=0.765)$, and MMSE$\operatorname{HMSE}(\mathrm{B}=37.245, \mathrm{SE}=15.624, p=0.140)$ were not significant predictors of LCPTLAS performance.

One-way analysis of variance revealed statistically significant differences and large effect sizes regarding not only the MMSE/HSME scores $[\mathrm{F}(5$, 119) $\left.=34.101, p<0.001, \eta^{2}=0.590\right]$, but also the financial capacity as examined by LCPTLAS for the six groups $\left[\mathrm{F}(5,115)=71.128, p<0.001, \eta^{2}=0.763\right]$ (Table 1).

In addition to the above analyses, a one-way analysis of variance (one-way ANOVA with bootstrapping) was performed. The procedure involved sampling with replacement ( 1,000 samples of 120 cases) from the original data. The results confirm the abovementioned statistically significant differences and are shown in Table 2.

\section{DISCUSSION}

These findings provide strong support for a noticeable financial incapacity in multiple-domain aMCI when illiteracy exists. The fact that performance in multiple-domain aMCI in illiterate individuals 
Table 2

Results from bootstrap estimations regarding LCPTLAS

\begin{tabular}{lccccc}
\hline LCPTLAS full total score & Statistic & Bias & Std. error & \multicolumn{2}{c}{$95 \%$ Confidence Interval } \\
\cline { 4 - 6 } & & & & Lower & Upper \\
\hline aMCI high education & 195.29 & -0.0165 & 1.2375 & 193.1502 & 197.8885 \\
aMCI low education & 142.31 & 0.2389 & 8.8108 & 126.2000 & 159.8664 \\
aMCI illiterate & 110.85 & -0.1079 & 3.4072 & 104.8130 & 117.9980 \\
HC high education & 211.55 & 0.0093 & 0.2240 & 211.0588 & 211.9545 \\
HC low education & 199.20 & 0.0490 & 3.9900 & 190.8650 & 206.2485 \\
HC illiterate & 195.25 & 0.2096 & 5.4852 & 183.4468 & 205.2607 \\
\hline
\end{tabular}

resembles more of mild AD performance (as indicated by the scores of mild AD patients in a previous study [8]), is considered as a novel finding. Our findings may have important implications for predictions of financial capacity performance in everyday settings as well as during the clinical examination.

The current study has some limitations, such as the small sample size, and the exclusion of patients with single-domain sub-type of aMCI (as a homogeneous sample regarding the diagnosis was desired). In addition to that, an overrepresentation of women among all groups should be noted as the older population in Greece consists mainly of women. It is of interest that in this study the majority of the sample is female and that women in previous studies have been reported to demonstrate poor mathematical skills, a finding that supports a hypothesized gender gap in their responses to competition, perceived selfworth, and not their actual arithmetical performance $[15,16]$. Thus, adequate numerical and mathematical skills (quantitative abilities) is a prerequisite to function effectively in daily life, and males and females perform similarly, regardless of stereotypes, in mathematics according to recent meta-analyses $[17,18]$. One last point that needs to be taken into consideration for the abovementioned findings is the way that the stratification-grouping was made for the illiterate (uneducated, never attended school) and the literate with low education and high education as there are many ways to differentiate the group of literate with low education from the group with high education. The way that these two groups were defined, followed a previous study in Greece. The literate with low education (up to 9 years of school attendance) and the literate with high education (more than 9 years of school attendance) [19].

This is the first study to show that literacy/illiteracy has a differentiating power on financial capacity performance in the group of multiple-domain aMCI patients. Although illiteracy seems to have a disastrous influence similar to the detrimental influence of depression on financial capacity in Parkinson's dis- ease and vascular dementia [2-4], further research should clarify the exact components of the influence of schooling background on neuropsychological test performance and how it can affect cognitive performance [20] as well as other variables such as prior financial experiences due to job before retirement, while the role of gender should be examined in larger samples of men as well as women. One more point that could be further investigated is the role not only of cognitive, but also emotional factors, since depression, anxiety, and apathy are common in MCI patients [21], and financial capacity has been found to be influenced by apathy more than depression in Parkinson's disease with dementia and frontotemporal dementia [22].

\section{CONFLICT OF INTEREST}

The authors have no conflict of interest to report.

\section{REFERENCES}

[1] Arce Rentería M, Vonk JMJ, Felix G, Avila JF, Zahodne LB, Dalchand E, Frazer KM, Martinez MN, Shouel HL, Manly JJ (2019) Illiteracy, dementia risk, and cognitive trajectories among older adults with low education. Neurology 93 , e2247-e2256.

[2] Giannouli V, Tsolaki M (2019) Depression and financial capacity assessment in Parkinson's disease with dementia: Overlooking an important factor? Psychiatriki 30, 66-70.

[3] Giannouli V, Tsolaki M (2021) Vascular dementia, depression and financial capacity assessment. Alzheimer Dis Assoc Disord 35, 84-87.

[4] Giannouli V, Tsolaki M (2020) Unraveling Ariadne's thread into the labyrinth of aMCI: Depression and financial capacity. Alzheimer Dis Assoc Disord, doi: 10.1097/WAD.0000000000000417.

[5] Wood SA, Liu PJ, Hanoch Y, Estevez-Cores S (2016) Importance of numeracy as a risk factor for elder financial exploitation in a community sample. J Gerontol B Psychol Sci Soc Sci 71, 978-986.

[6] Fountoulakis KN, Kaprinis SG, Kaprinis GS (2002) Special characteristics affecting the neuropsychologic assessment of the elderly in Greece. Am J Alzheimers Dis Other Demen 17, 273-276. 
[7] Kosmidis MH (2018) Challenges in the neuropsychological assessment of illiterate older adults. Lang Cogn Neurosci 33, 373-386.

[8] Griffith HR, Belue K, Sicola A, Krzywanski S, Zamrini E, Harrell L, Marson DC (2003) Impaired financial abilities in mild cognitive impairment: A direct assessment approach. Neurology 60, 449-457.

[9] Niccolai LM, Triebel KL, Gerstenecker A, McPherson TO, Cutter GR, Martin RC, Marson DC (2017) Neurocognitive predictors of declining financial capacity in persons with mild cognitive impairment. Clin Gerontol 40, 14-23.

[10] Giannouli V, Tsolaki M (2019) Are left angular gyrus and amygdala volumes important for financial capacity in mild cognitive impairment? Hell J Nucl Med 22, 160-164.

[11] Petersen RC, Roberts RO, Knopman DS, Boeve BF, Geda YE, Ivnik RJ, Smith GE, Jack CR Jr (2009) Mild cognitive impairment: Ten years later. Arch Neurol 66, 1447-1455.

[12] Fountoulakis KN, Tsolaki M, Iacovides A, Yesavage J, O'Hara R, Kazis A, Ierodiakonou C (1999) The validation of the short form of the Geriatric Depression Scale (GDS) in Greece. Aging Clin Exp Res 11, 367-372.

[13] Giannouli V, Stamovlasis D, Tsolaki M (2018) Exploring the role of cognitive factors in a new instrument for elders' financial capacity assessment. J Alzheimers Dis 62, 15791594.

[14] Tsolaki M, Iakovidou V, Navrozidou H, Aminta M, Pantazi T, Kazis A (2000) Hindi Mental State Examination (HMSE) as a screening test for illiterate demented patients. Int J Geriatr Psychiatry 15, 662-664.
[15] Niederle M, Vesterlund L (2010) Explaining the gender gap in math test scores: The role of competition. J Econ Perspect 24, 129-144.

[16] Thompson T, Dinnel DL (2007) Poor performance in mathematics: is there a basis for a self-worth explanation for women? Educ Psychol 27, 377-399.

[17] Lindberg SM, Hyde JS, Petersen JL, Linn MC (2010) New trends in gender and mathematics performance: a metaanalysis. Psychol Bull 136, 1123.

[18] Halpern DF, Benbow CP, Geary DC, Gur RC, Hyde JS, Gernsbacher MA (2007) The science of sex differences in science and mathematics. Psychol Sci Public Interest 8, 151.

[19] Kosmidis MH, Tsapkini K, Folia V (2006) Lexical processing in illiteracy: effect of literacy or education? Cortex $\mathbf{4 2}$, 1021-1027.

[20] Giannouli V, Tsolaki M (2014) Legal capacity of the elderly in Greece. Hell J Nucl Med 17, 2-6.

[21] Ma L (2020) Depression, anxiety, and apathy in mild cognitive impairment: current perspectives. Front Aging Neurosci $12,9$.

[22] Giannouli V, Tsolaki M (2021) Is depression or apathy playing a key role in predicting financial capacity in Parkinson's disease with dementia and frontotemporal dementia? Brain Sci 11, 785 . 